Network Working Group

Request for Comments: 2513

Category: Standards Track
K. McCloghrie Cisco Systems, Inc.

J. Heinanen Telia Finland, Inc. W. Greene MCI Telecommunications Corp. A. Prasad

Cisco Systems, Inc. February 1999

\title{
Managed Objects for Controlling the Collection and Storage of Accounting Information for Connection-Oriented Networks
}

\section{Status of this Memo}

This document specifies an Internet standards track protocol for the Internet community, and requests discussion and suggestions for improvements. Please refer to the current edition of the "Internet Official Protocol Standards" (STD 1) for the standardization state and status of this protocol. Distribution of this memo is unlimited.

Copyright Notice

Copyright (C) The Internet Society (1999). All Rights Reserved.

Table of Contents

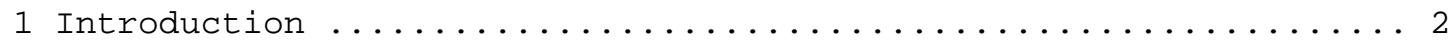

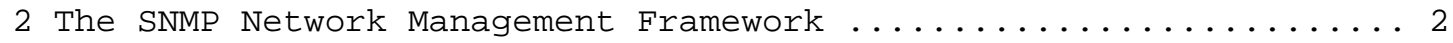

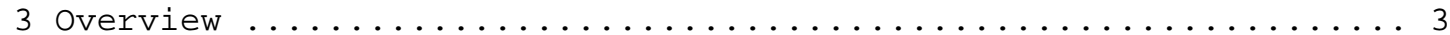

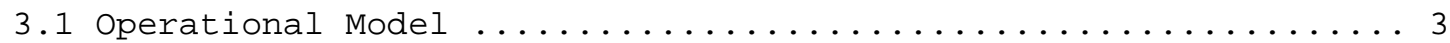

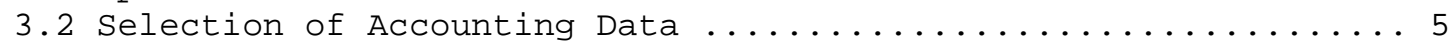

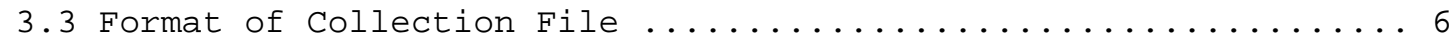

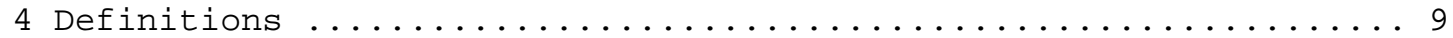

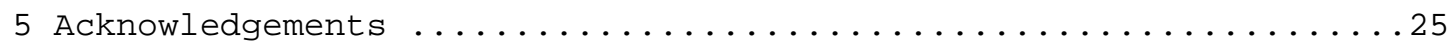

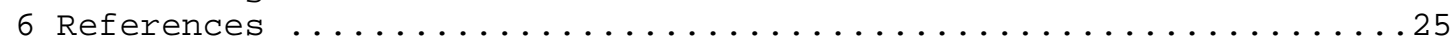

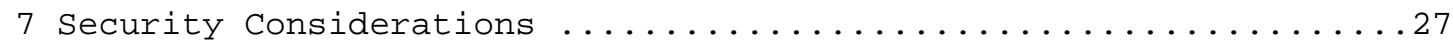

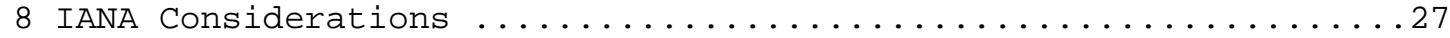

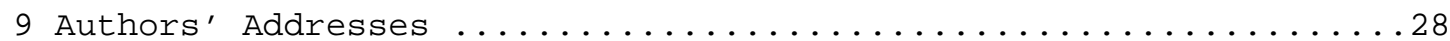

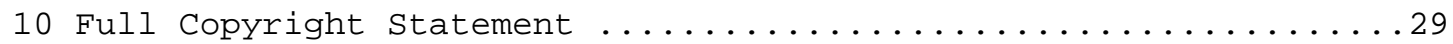




\section{Introduction}

This memo defines a portion of the Management Information Base (MIB) for use with network management protocols in the Internet community. In particular, it describes managed objects used for controlling the collection and storage of accounting information for connectionoriented networks such as ATM. The accounting data is collected into files for later retrieval via a file transfer protocol. For information on data which can be collected for ATM networks, see [19].

2. The SNMP Network Management Framework

The SNMP Management Framework presently consists of five major components:

o An overall architecture, described in RFC 2271 [1] .

- Mechanisms for describing and naming objects and events for the purpose of management. The first version of this structure of Management Information (SMI) is called SMIV1 and described in $\operatorname{STD} 16, \operatorname{RFC} 1155$ [2], STD 16, RFC 1212 [3] and RFC 1215 [4]. The second version, called SMIv2, is described in RFC 1902 [5], $\mathrm{RFC} 1903$ [6] and RFC 1904 [7].

- Message protocols for transferring management information. The first version of the SNMP message protocol is called SNMPV1 and described in STD 15, RFC 1157 [8]. A second version of the SNMP message protocol, which is not an Internet standards track protocol, is called SNMPv2c and described in RFC 1901 [9] and RFC 1906 [10]. The third version of the message protocol is called SNMPv3 and described in RFC 1906 [10], RFC 2272 [11] and RFC 2274 [12].

- Protocol operations for accessing management information. The first set of protocol operations and associated PDU formats is described in STD 15, RFC 1157 [8]. A second set of protocol operations and associated PDU formats is described in RFC 1905 [13].

- A set of fundamental applications described in RFC 2273 [14] and the view-based access control mechanism described in RFC 2275 [15].

Managed objects are accessed via a virtual information store, termed the Management Information Base or MIB. Objects in the MIB are defined using the mechanisms defined in the SMI. 
This memo specifies a MIB module that is compliant to the SMIv2. A MIB conforming to the SMIv1 can be produced through the appropriate translations. The resulting translated MIB must be semantically equivalent, except where objects or events are omitted because no translation is possible (e.g., use of Counter64). Some machine readable information in SMIV2 will be converted into textual descriptions in SMIv1 during the translation process. However, this loss of machine readable information is not considered to change the semantics of the MIB.

\section{Overview}

In some connection-oriented network environments, there is a need for the network administrator to be able to collect accounting data on the usage of bandwidth/resources by connections (e.g., ATM connections) within the network. Data collection should be available for switched virtual connections (SVCs and SVPs), and permanent virtual connections (PVCs and PVPS), including soft-permanent virtual connections (SPVCCs and SPVPCs). This need exists for ATM networks, and may well exist for other connection-oriented networks, such as Frame Relay.

The potential quantity of such accounting information is such that it is not, in general, feasible to retrieve the information via SNMP. A better method is to store the collected accounting information in a file which can be subsequently retrieved via a file transfer protocol.

It is, however, appropriate to provide management control of the selection and collection of such accounting data via SNMP. This memo describes a MIB module which provides such control in a manner independent of the type of network. One or more other documents provide definitions of particular items of accounting data which can be selected; for example, a particular set of data items which can be collected for ATM networks is specified in [19].

\subsection{Operational Model}

The requirement is for switches (e.g., ATM switches) to collect data concerning the connections which are routed across some subset of their interfaces (e.g., ATM UNI and/or NNI interfaces). The collected data is stored into one or more "files". The use of multiple files allows, for example, the data collected for PVCs to be different from that collected for SVCs.

In order to retrieve the data currently being stored in a file, the administrator instructs the switch to terminate the collection of data into that file, and start collecting data into a new file. 
After this operation, the data in the old file is available for retrieval via file transfer.

A collection file is defined to have a maximum size. When the size of the file currently being collected exceeds a threshold percentage of that maximum size, an SNMP notification (e.g., a trap) can be optionally generated. An SNMP notification might also be generated if the file reaches its maximum size.

The accounting data collected for each connection consists of a set of objects and their values. The set of objects and their values are collected on one or more of the following occasions:

(1) on the release (termination) of a connection optionally including failed connection attempts;

(2) for each active connection (having a particular minimum age) on a periodic basis;

(3) for each active connection (having a particular minimum age) when so commanded by a management application.

While collecting data to be stored in a particular file, the same set of objects is collected for each connection on each occasion. Having the same set of objects stored on each occasion allows the optimization of storing only the values of those objects. This results in a significantly smaller file size, since it allows the names of the objects to be stored once and only once at the beginning of the file, rather than having to store every value as a (name, value) pair.

Two modes of agent behaviour are allowed on the event of a file reaching its maximum size:

(1) management application in control:

The agent does not automatically swap to a new file; rather, it discards newly collected data until the management application subsequently instructs it to swap to a new file. Before swapping to a new file, the name of the file into which data is currently being collected is an implementation issue of no concern to an NM application; after swapping to a new file, the name of the file available for retrieval is as specified by the controlling MIB objects. This behaviour allows the application to know exactly how many files need to be retrieved and their names without having to perform any type of file directory operation, but also results in the possibility that data will be discarded if the application does not instruct the agent to swap 
within the required time frame.

agent automatically swaps to new file:

The agent terminates collection into the current (full) file, and begins collecting data into a new version of the same base file name. This behaviour aims to avoid loss of data by assuming that additional storage space is actually available to create a new version of the file. To support this behaviour, files are named using suffixes, such that when the current version of the file becomes full, the agent begins collecting data into a file with the same base file-name but with an incremented (or otherwise modified) suffix. This requires the application to perform file directory operations prior to retrieving completed files in order to know how many and which suffixes have been used.

With either behaviour, any completed file must be an integral number of connection records (see below). When a file reaches its maximum size, collection into that file is terminated either immediately before or immediately after storing the whole of the current connection record into the file. The former causes the file to be just less than its maximum size, and the latter causes the file to be just greater than its maximum size.

\subsection{Selection of Accounting Data}

The items of accounting data to be collected are specified as a set of objects. Which objects are contained in such a set is selectable by an administrator through the specification of one or more (subtree, list) tuples, where the set of objects to be collected is the union of the subsets specified by each tuple:

'subtree' specifies an OBJECT IDENTIFIER value such that every object in the subset is named by the subtree's value appended with a single additional sub-identifier.

'list' specifies an OCTET STRING value, such that if the N-th bit of the string's value is set then the the subset contains the object named by appending $\mathrm{N}$ as a single additional subidentifier to the subtree.

The rationale for defining each subset as a (subtree, list) tuple is that one and only one OBJECT IDENTIFIER and one OCTET STRING is needed to define the subset of objects. This simplifies the MIB mechanisms needed for selection: an NM application needs to create only one conceptual row in a MIB table for each subset (rather than needing to create a conceptual row in a table for each and every 
object in the set).

The number of tuples supported by a particular switch is an implementation choice. One possibility is to support two (subtree, list) tuples so that one such tuple can specify a standard 'subtree' (e.g., the atmAcctngDataobjects subtree defined in [19]), and the second tuple can specify an enterprise-specific 'subtree'; this would allow the selected set of objects to be the union of a set of standard objects and a set of enterprise-defined objects.

\subsection{Format of Collection File}

A collection file generated by this process contains the values of MIB objects defined using the SMIv2. The standard way to encode the values of SNMP MIB objects in a device-independent manner is through the use of ASN.1's Basic Encoding Rules (BER) [18]. Thus, the standard format of an accounting file is defined here using the same adapted subset of ASN.1 [17] as the SMIV2.

The file consists of a set of header information followed by a sequence of zero or more collection records. The header information identifies (via sysName [16]) the switch which collected the data, the date and time at which the collection in to this file started, and the sequence of one or more (subtree, list) tuples identifying the objects whose values are contained in each connection record. The header information also includes a textual description of the data contained in the file.

Each connection record contains a sequence of values for each identified tuple, in the same order as the tuples are identified in the header information. For each tuple, the sequence of values are in ascending order of the sub-identifier which identifies them within the subtree.

Formally, an accounting file is an ASN.1 value with the following syntax:

File : : =

[1]

IMPLICIT SEQUENCE \{

sysName

- header information Displaystring, -- name of the switch

description

-- textual description of the collection Displaystring, startTime -- start time of the collection 


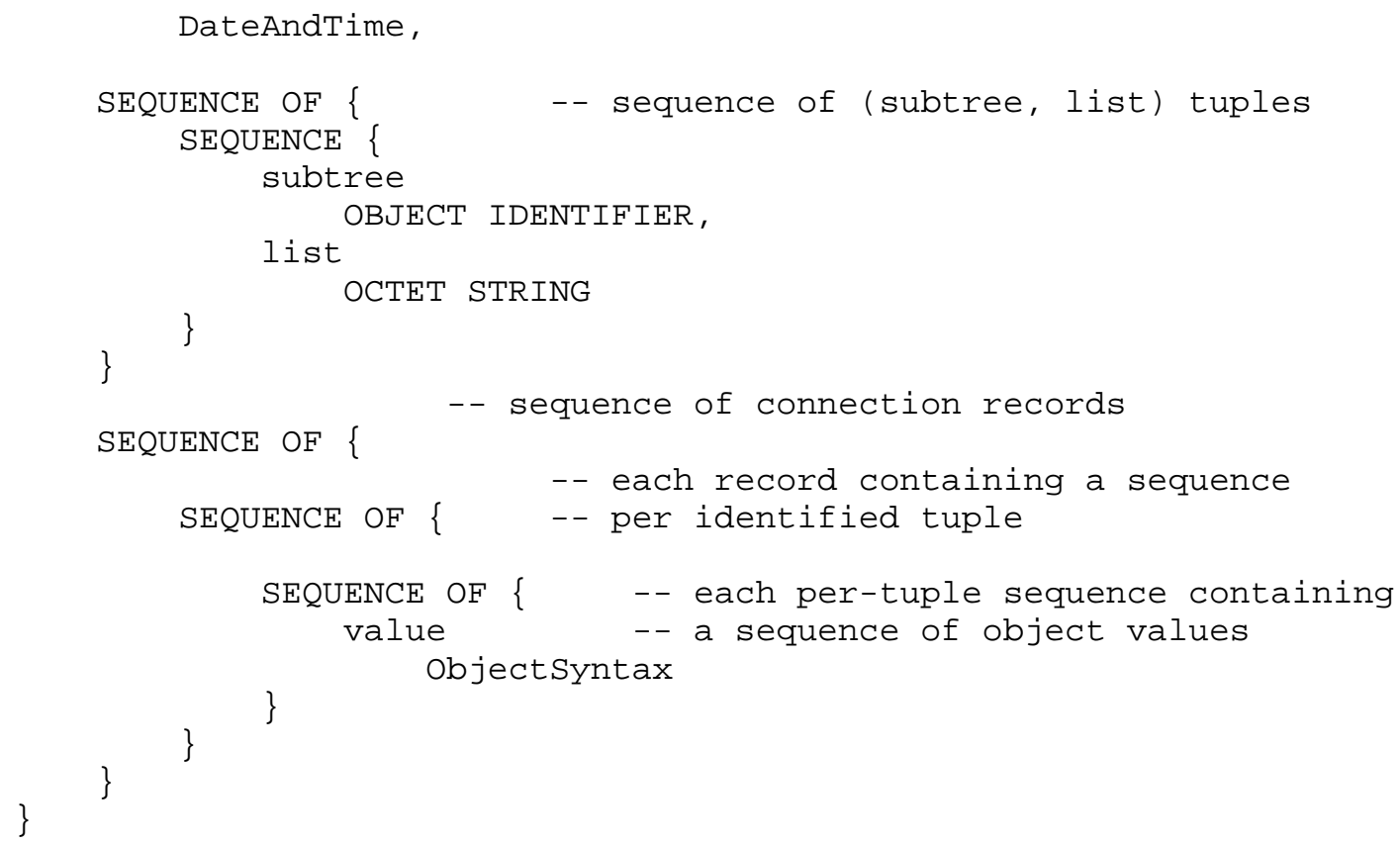

where:

(1) the value of the sysName component is that of the sysName object in the system group [16].

(2) each (subtree, list) specifies the set of objects contained in that tuple's sequence within each and every connection record.

(3) the tuples' sequences within each connection record occur in the same order as the (subtree, list) tuples occur in the header information.

(4) the object values within each connection record occur in the same order as they are represented by the bits in the corresponding list value.

(5) Objectsyntax is defined by the SMIv2 [5] .

(6) One particular category of object values deserves special attention: an object defined to hold the checksum value of an accounting record (e.g., atmAcctngRecordCrc16, defined in [19]). An object in this category will generally have a SYNTAX of a fixed-length OCTET STRING, and have its value initialized to the string of all zeros when composing the accounting record containing it, with the location of these zeros being saved. 
Once the record is generated, the checksum is calculated over the whole connection record (including the starting SEQUENCE OF and the trailing end-of-contents octets, if used), and then the zeros are overwritten (at the saved location) by the calculated value of the checksum.

The encoding of the above syntax using the Basic Encoding Rules is the same as defined by the SNMPv2 [10], with the following exception:

- when encoding the length field for a structured type, i.e., a SEQUENCE or SEQUENCE OF, the indefinite form encoding is permitted.

For example, the file containing the data:

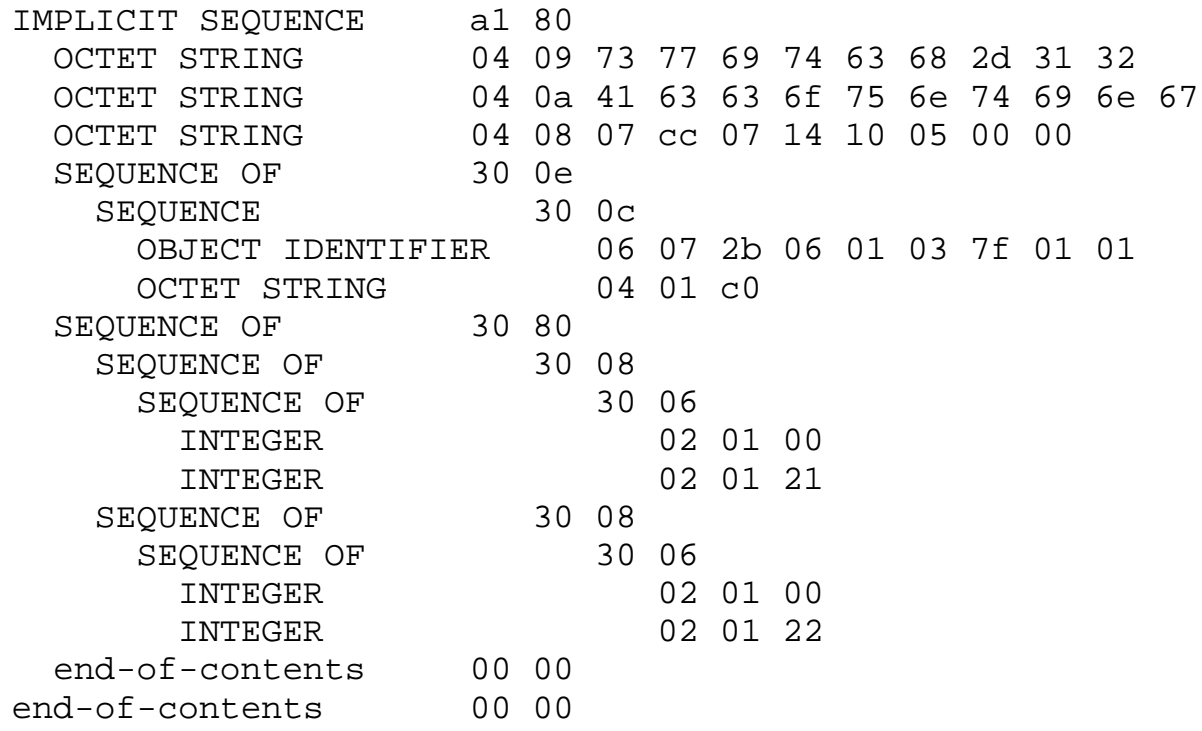

McCloghrie, et. al. Standards Track [Page 8] 
appended with a single
SYNTAX OBJECT IDENTIFIER

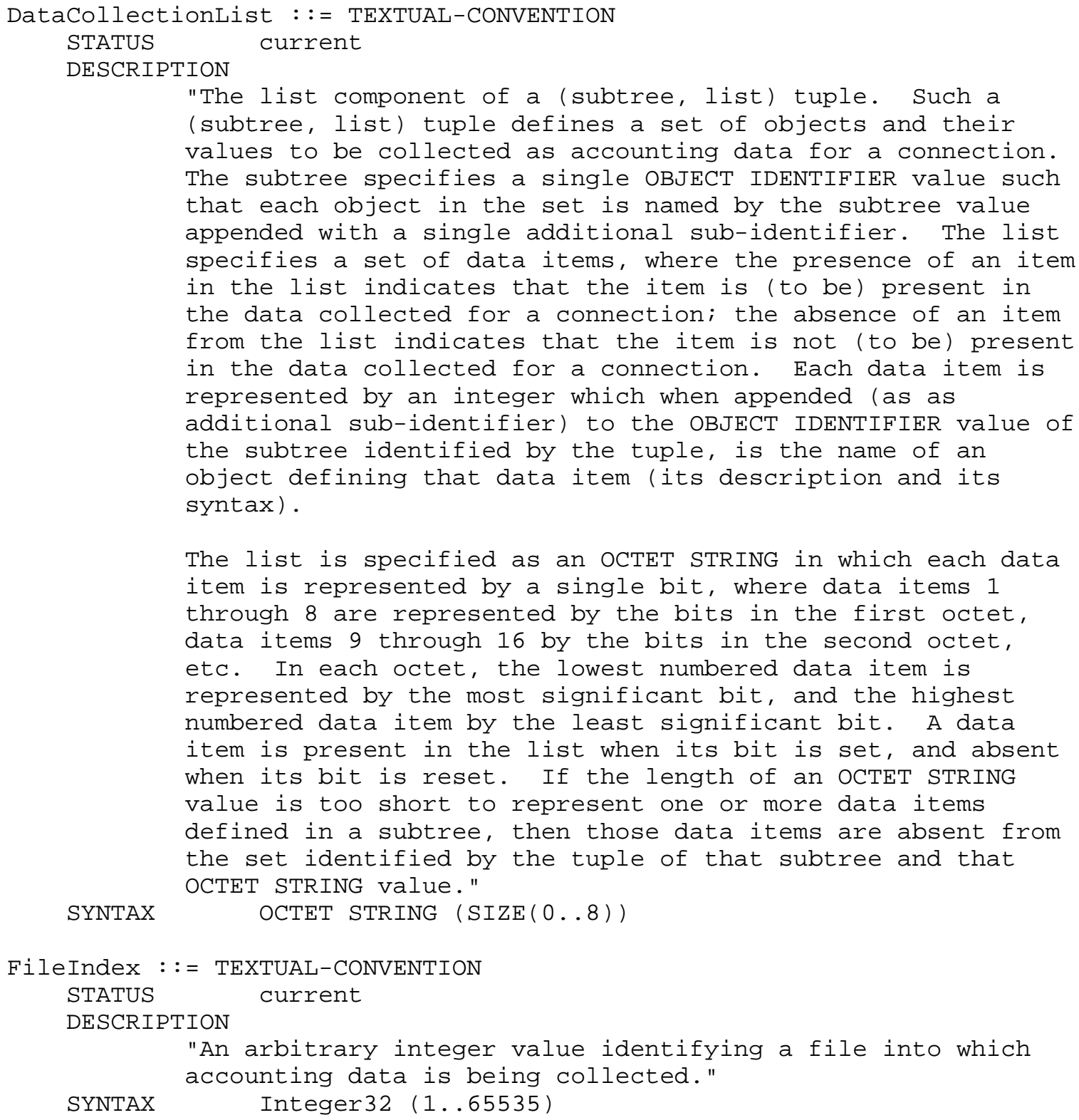




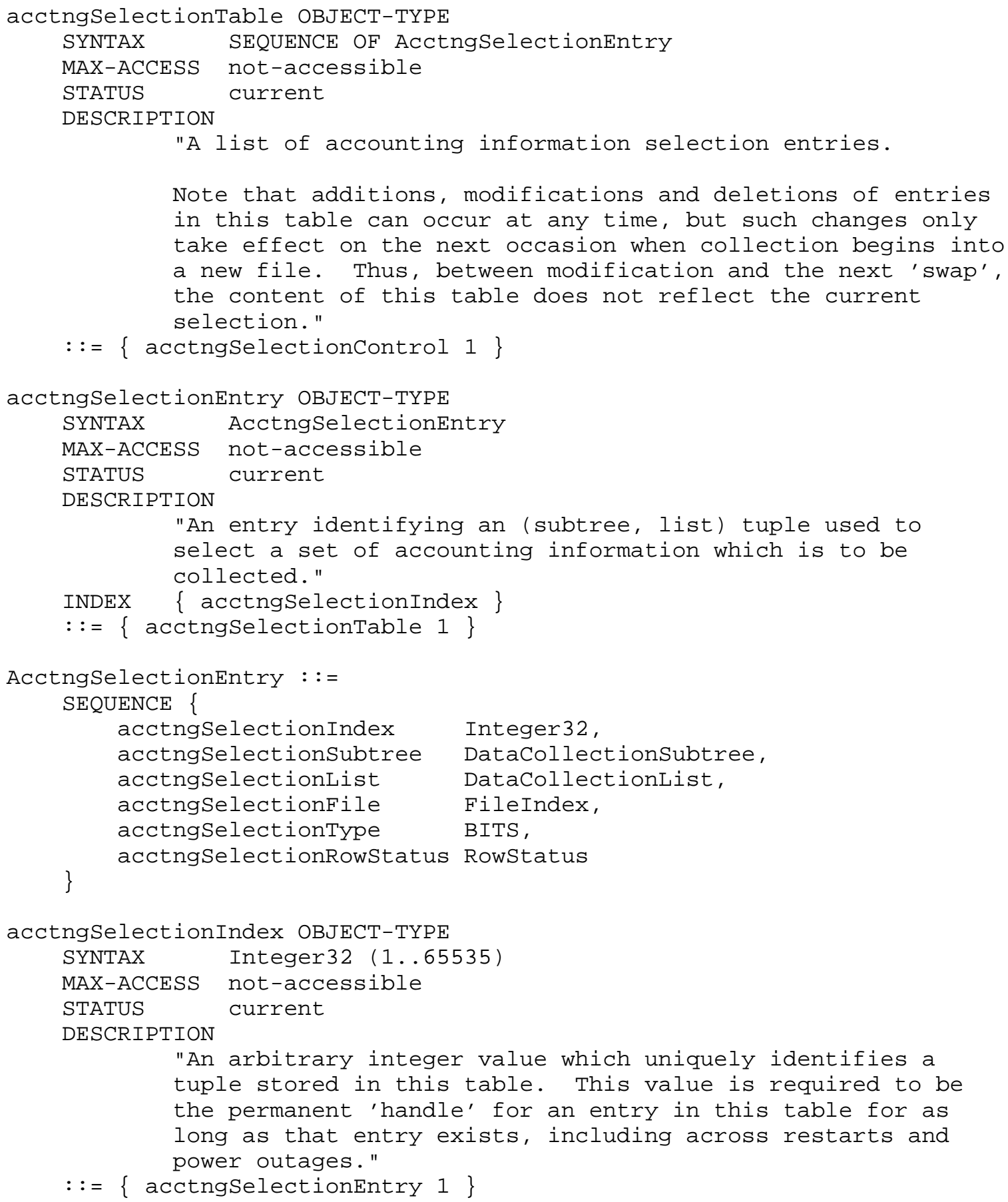




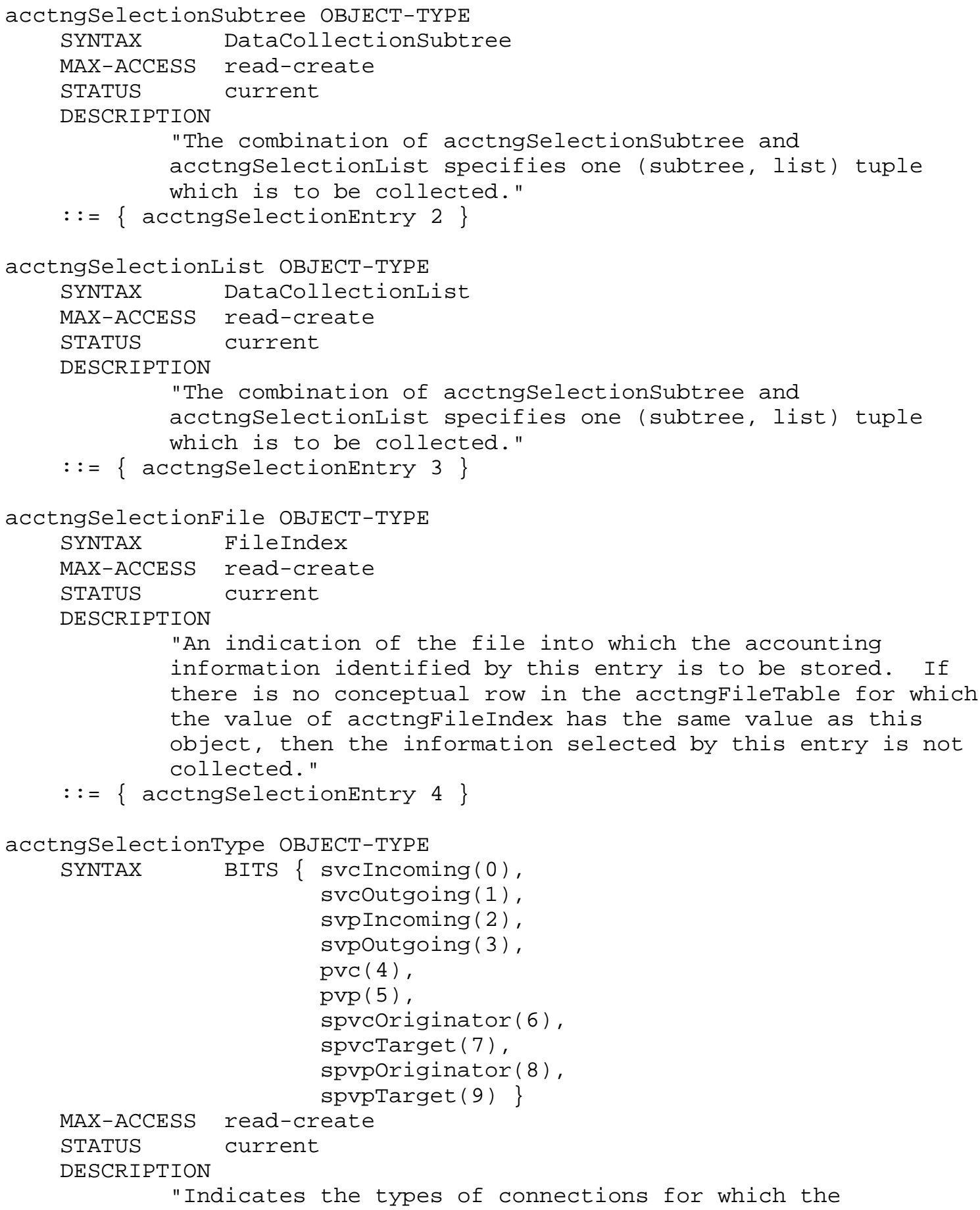

McCloghrie, et. al. Standards Track [Page 12] 


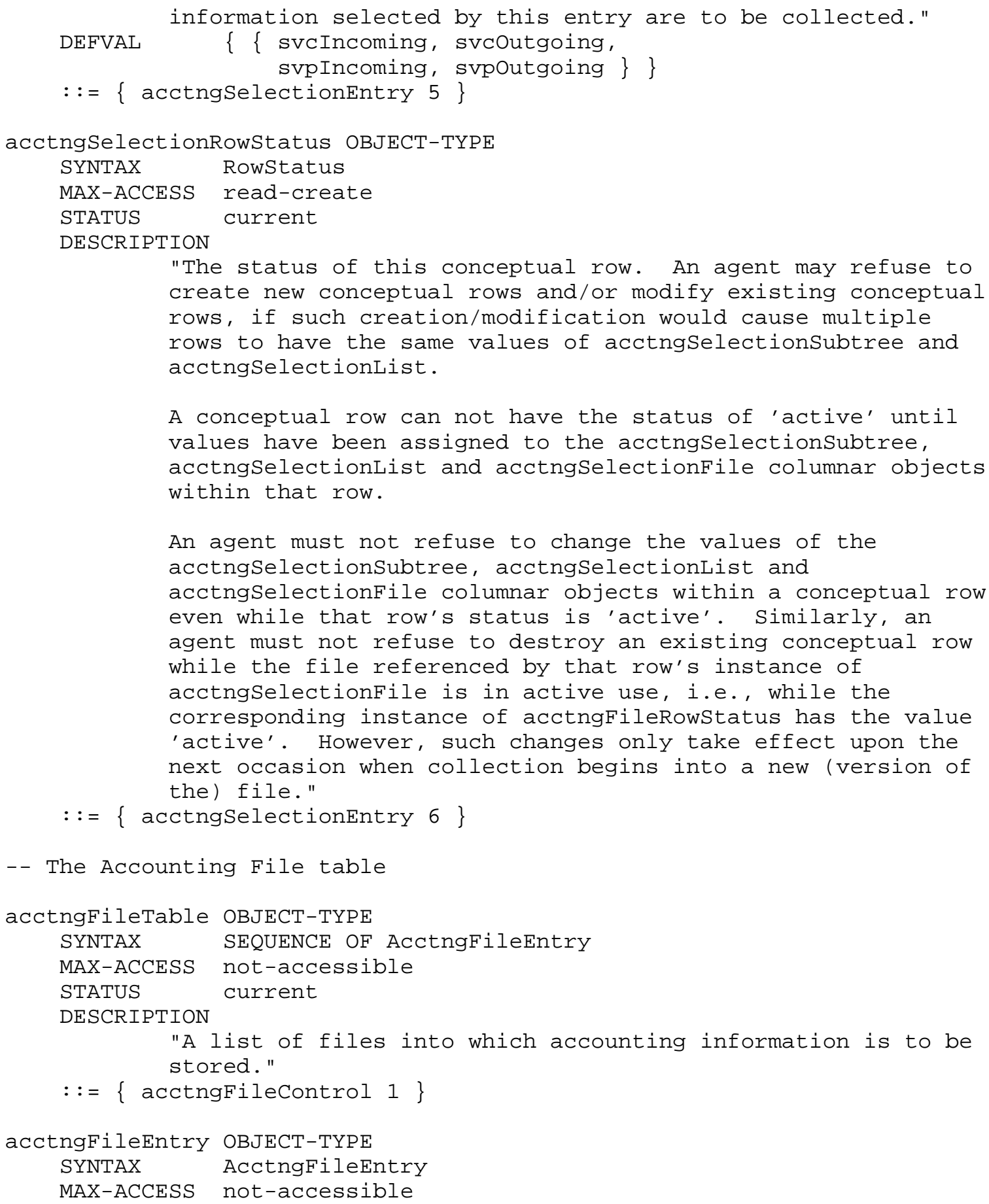




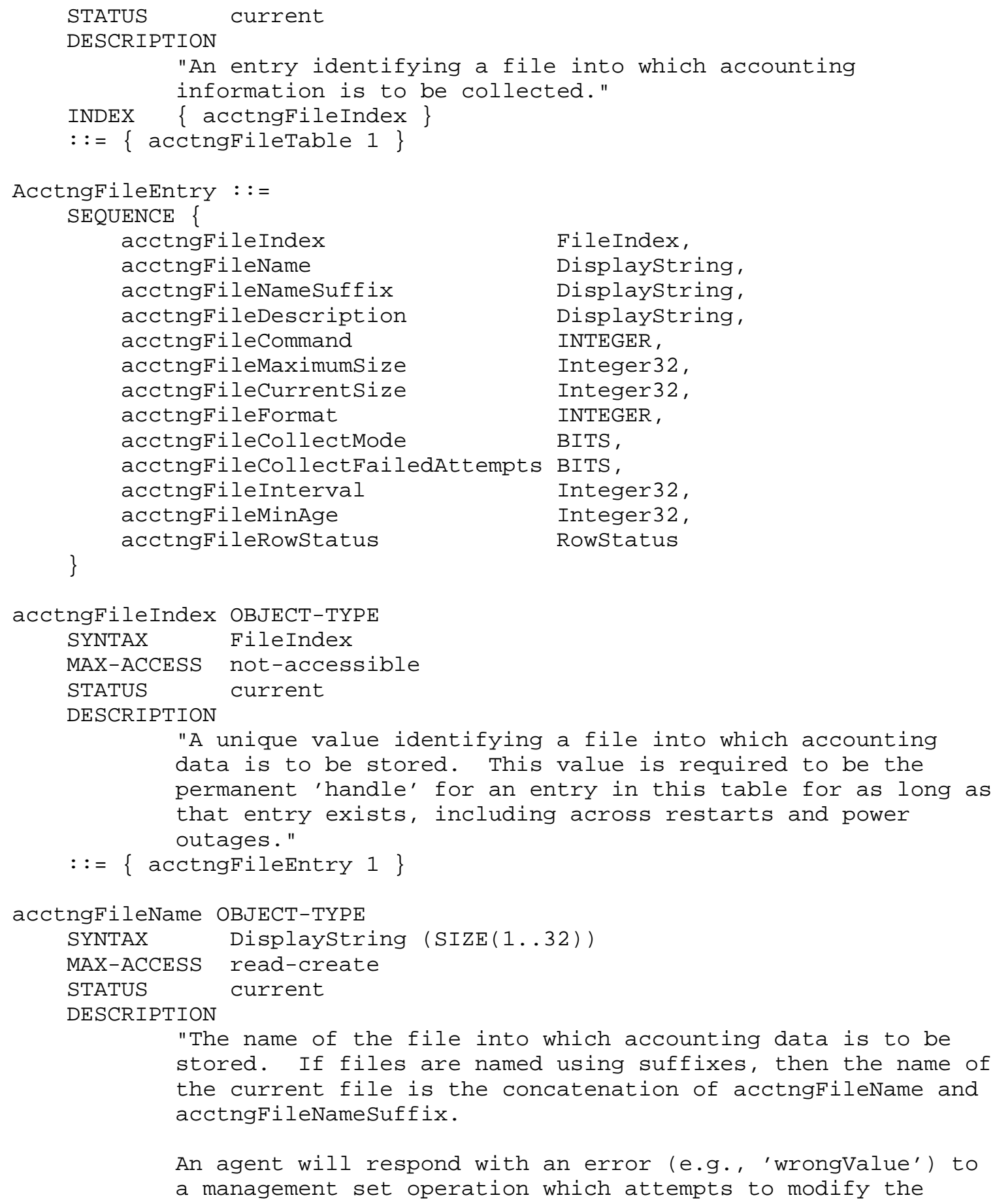




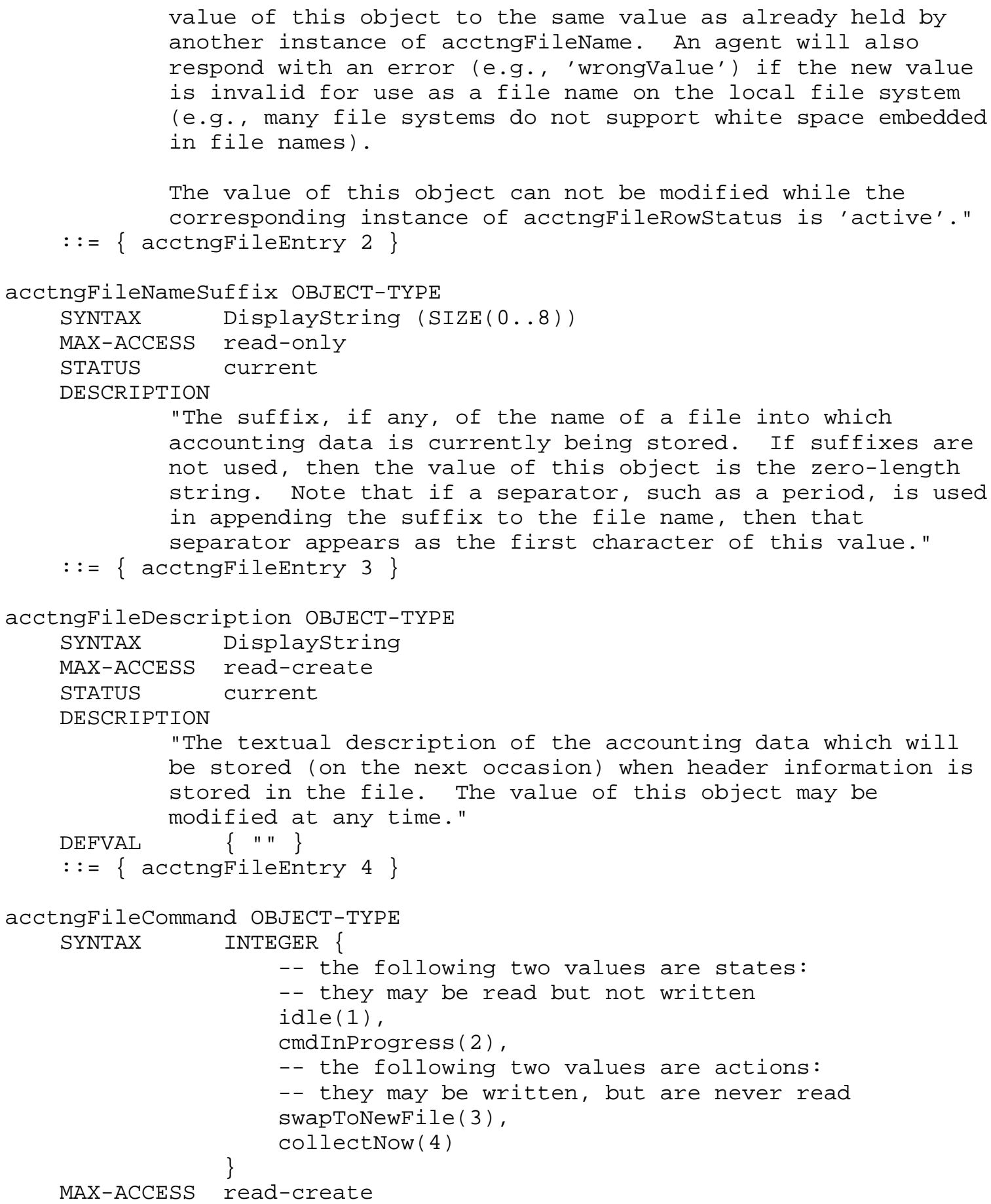

McCloghrie, et. al. Standards Track [Page 15] 


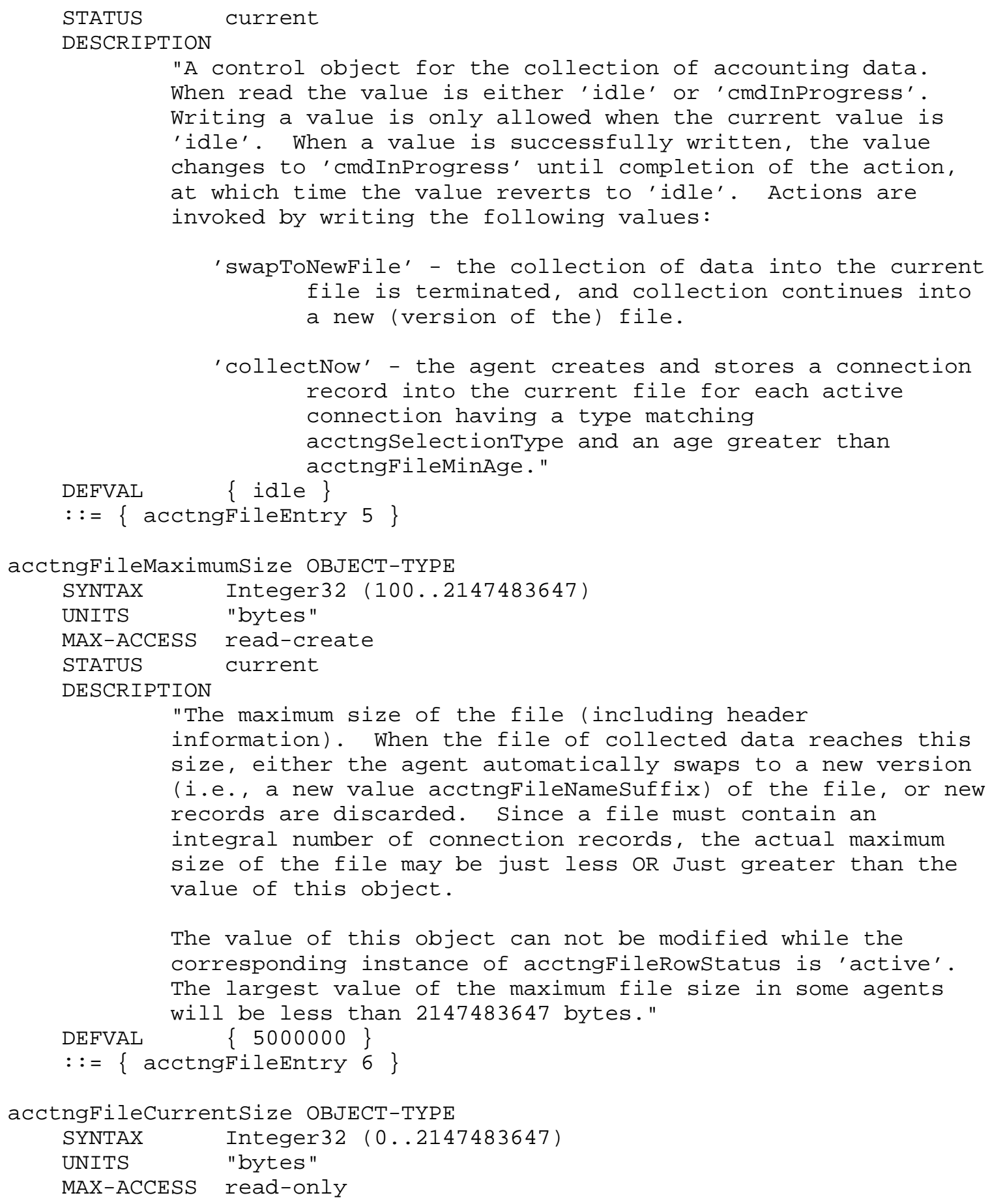




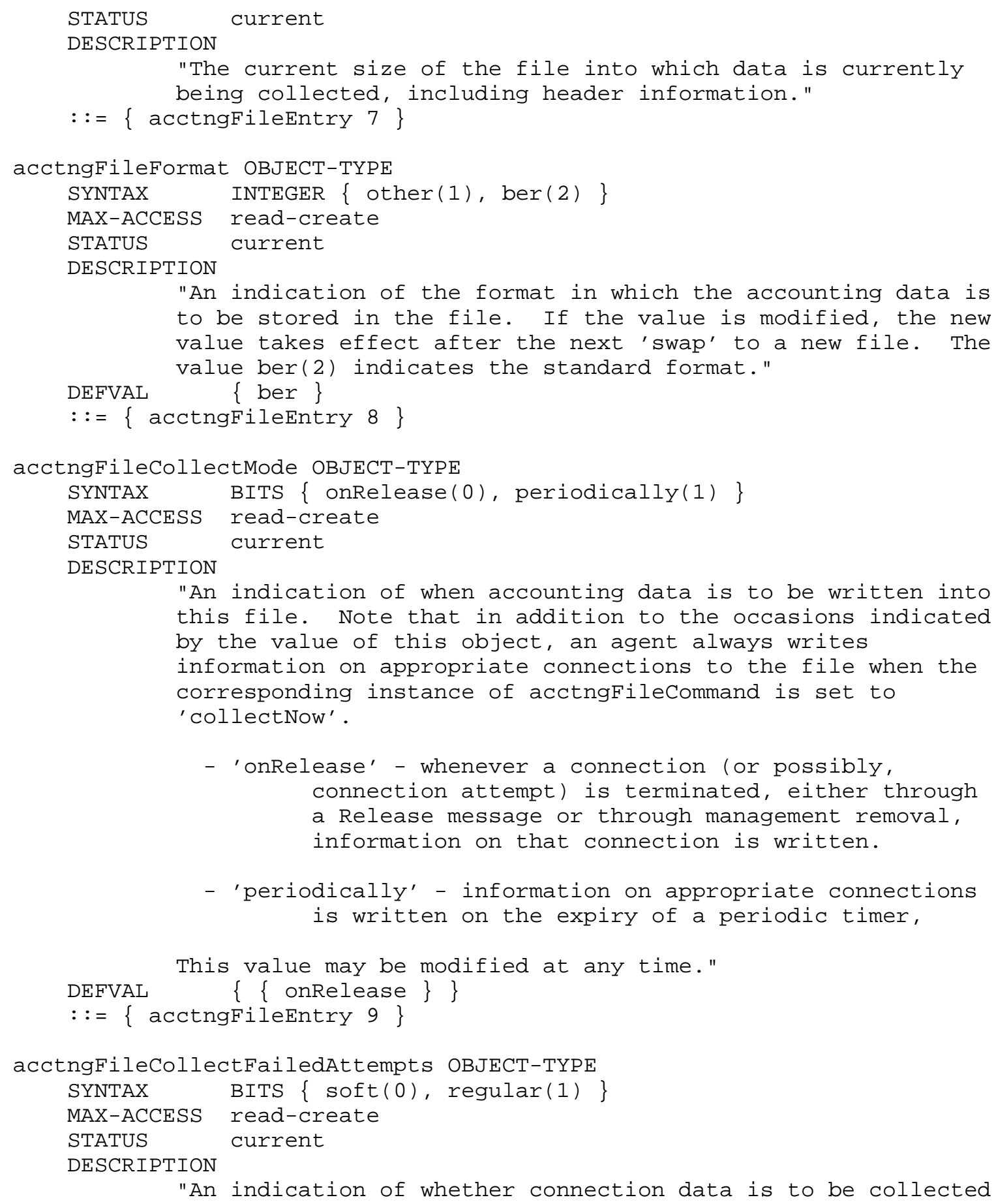




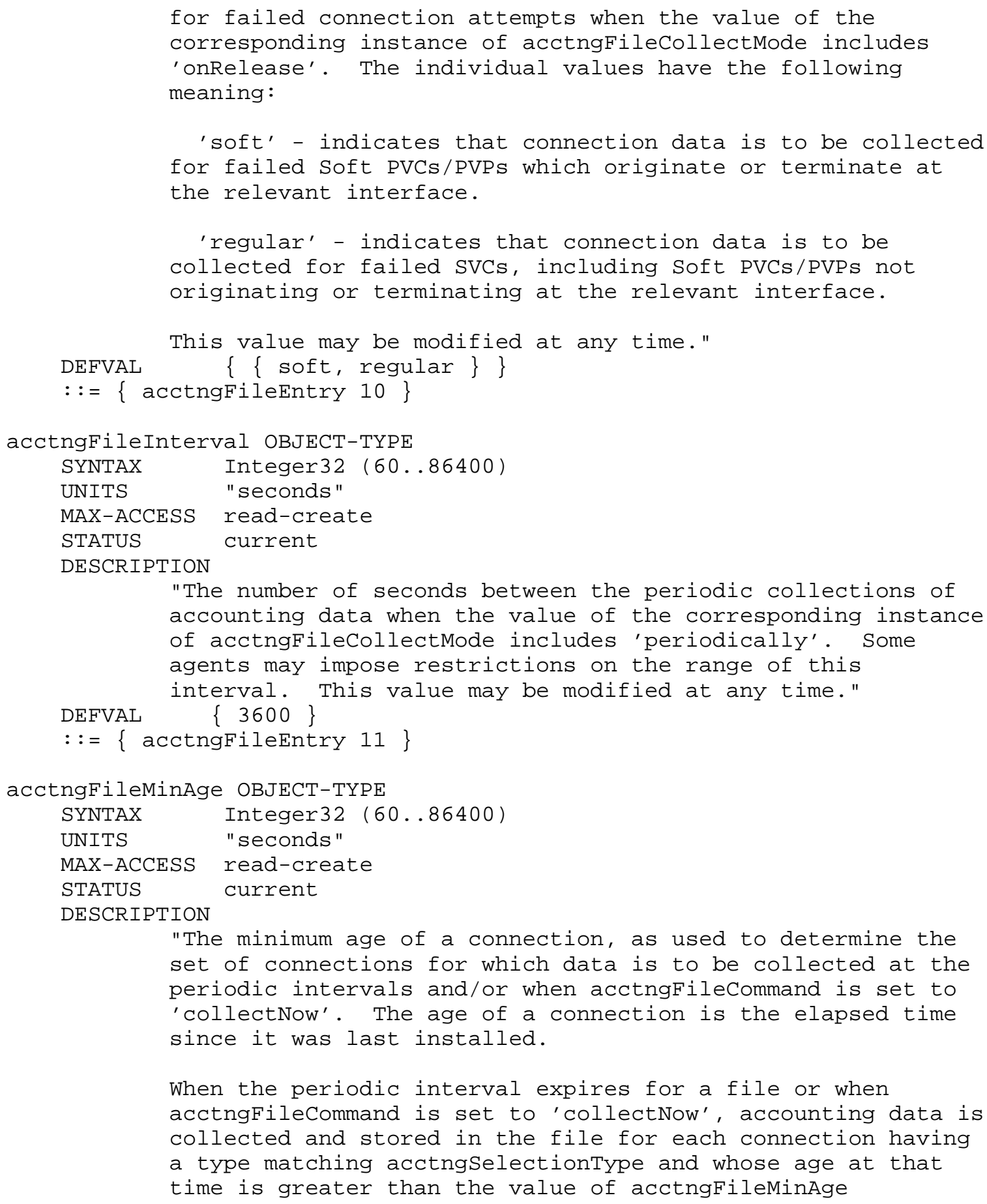




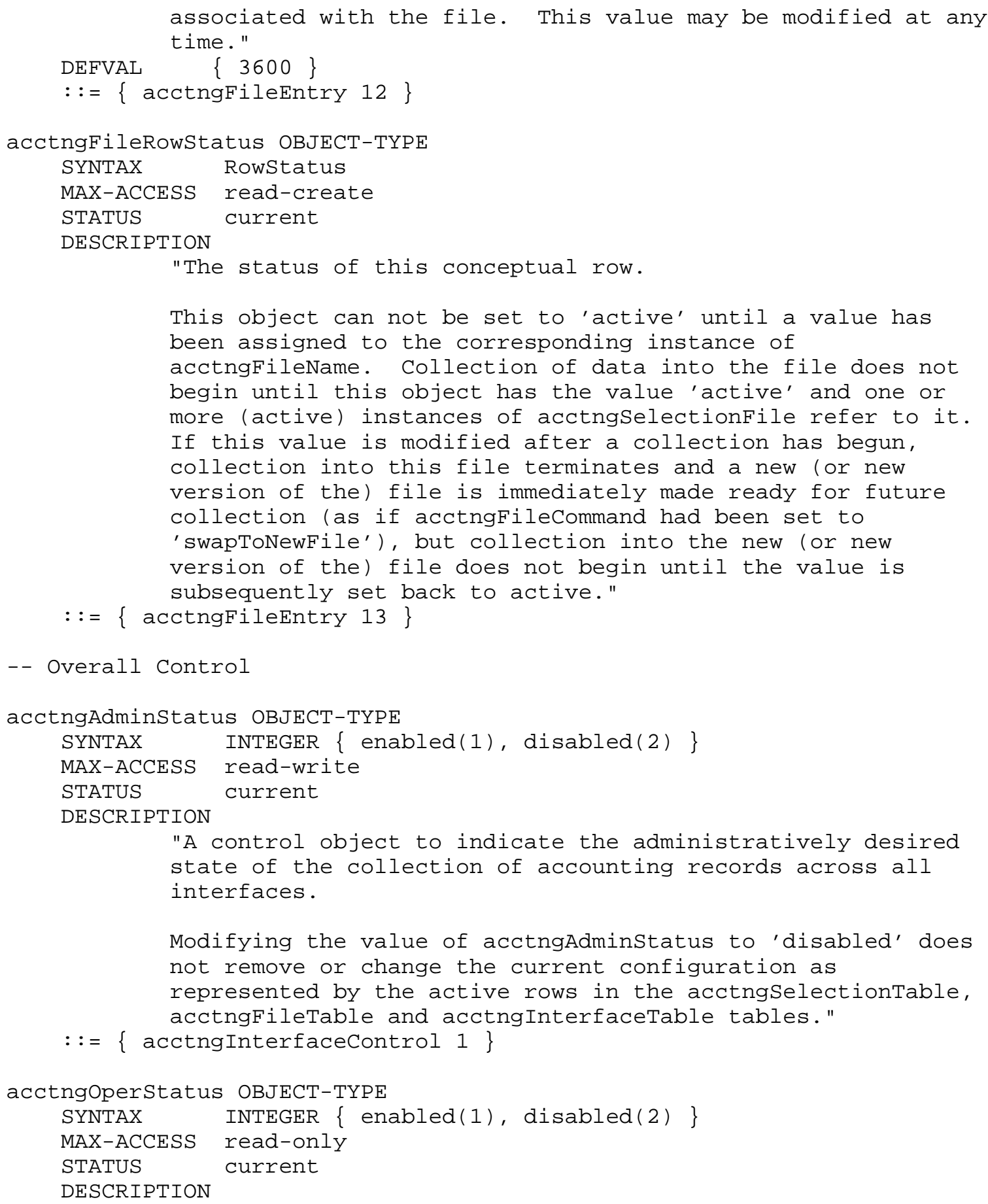




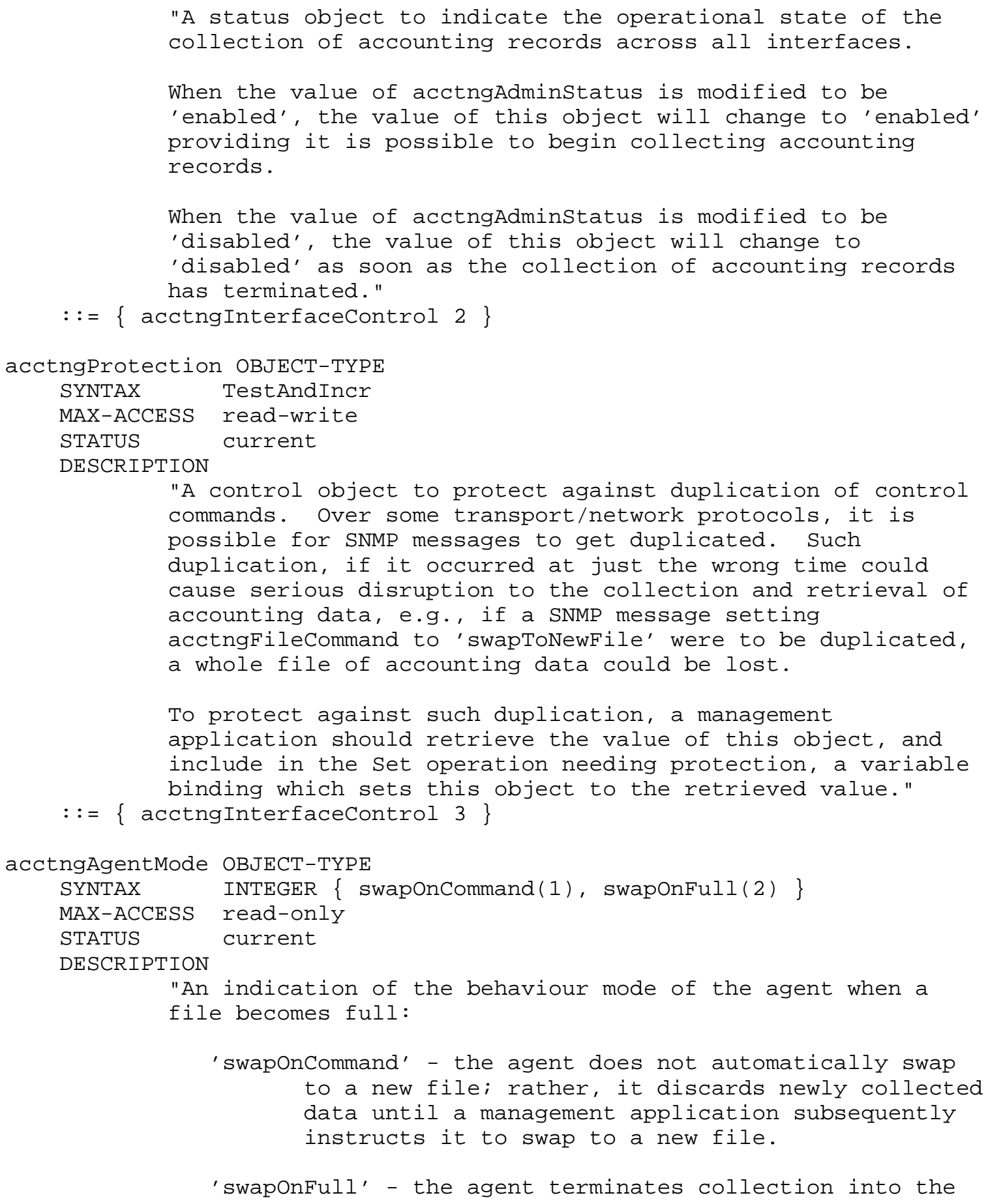




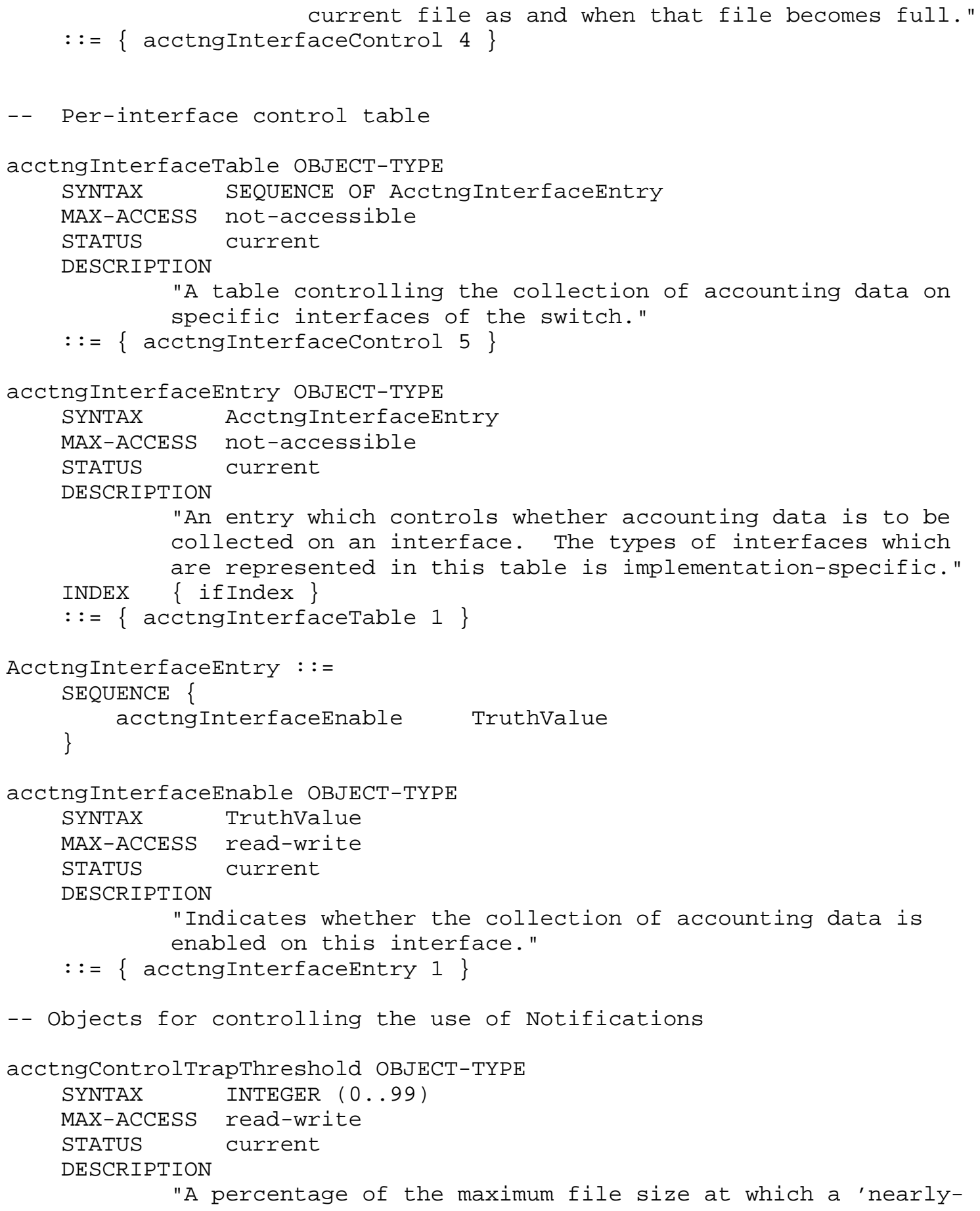




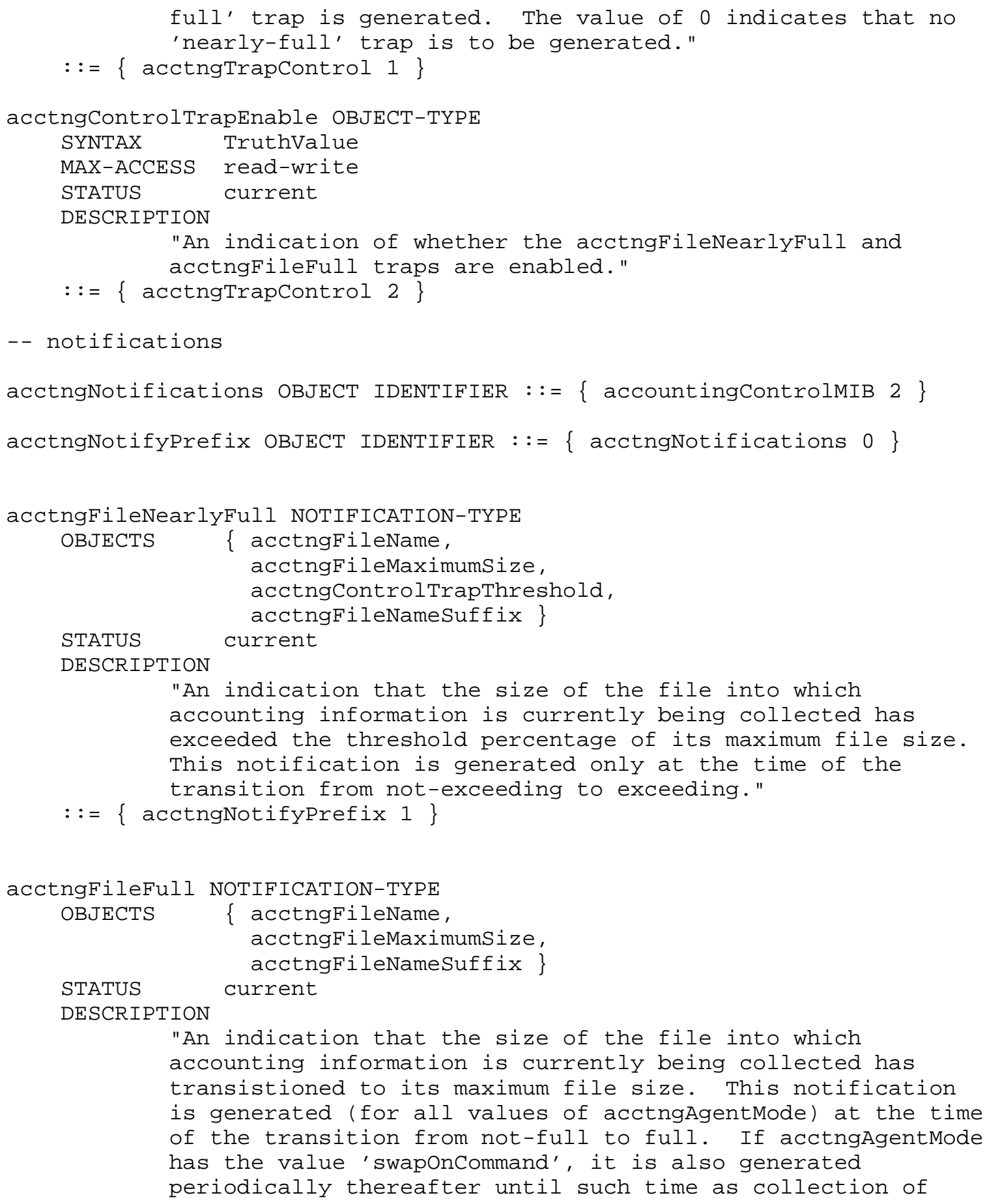




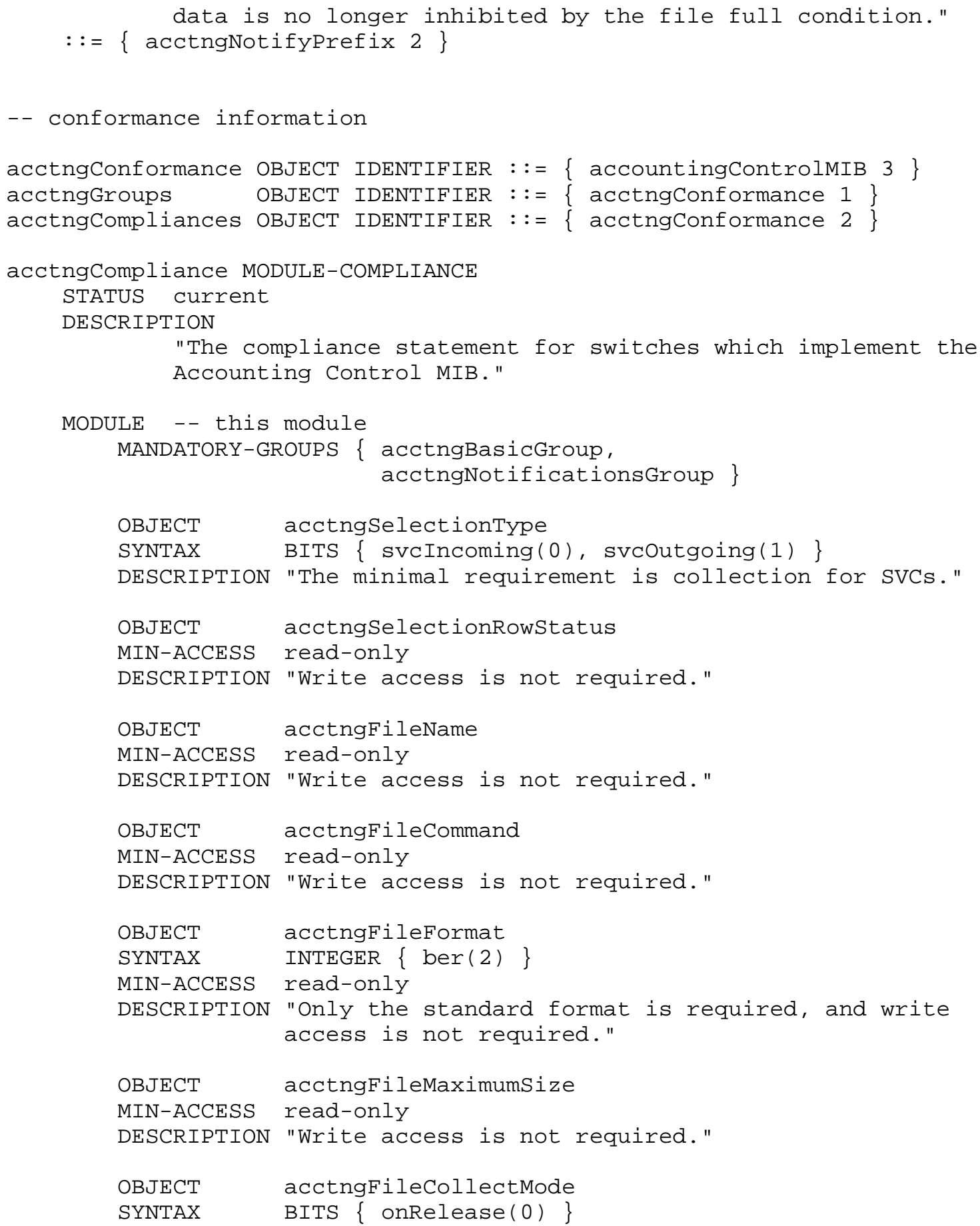




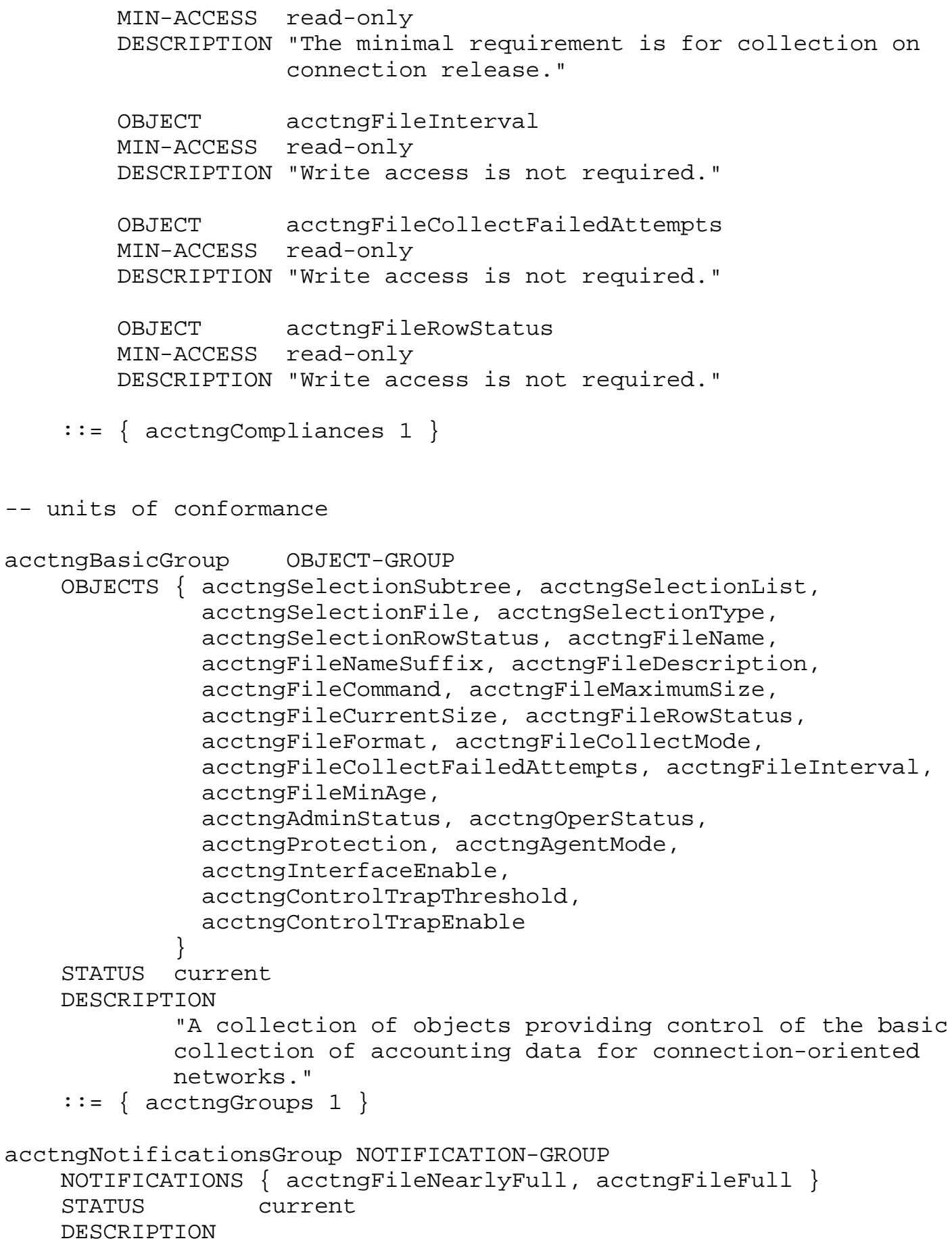




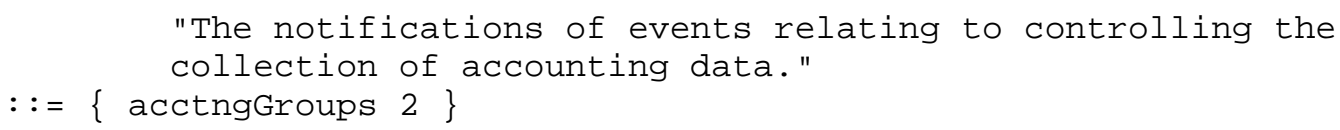

END

5. Acknowledgements

The comments of the IETF's AToM MIB Working Group are acknowledged.

6. References

[1] Harrington, D., Presuhn, R. and B. Wijnen, "An Architecture for Describing SNMP Management Frameworks", RFC 2271, January 1998.

[2] Rose, M. and K. McCloghrie, "Structure and Identification of Management Information for TCP/IP-based Internets", STD 16, RFC 1155, May 1990.

[3] Rose, M. and K. McCloghrie, "Concise MIB Definitions", STD 16, RFC 1212, March 1991.

[4] Rose, M., "A Convention for Defining Traps for use with the SNMP", RFC 1215, March 1991.

[5] SNMPv2 Working Group, Case, J., McCloghrie, K., Rose, M. and S. Waldbusser, "Structure of Management Information for Version 2 of the Simple Network Management Protocol (SNMPv2)", RFC 1902, January 1996.

[6] SNMPv2 Working Group, Case, J., McCloghrie, K., Rose, M. and S. Waldbusser, "Textual Conventions for Version 2 of the Simple Network Management Protocol (SNMPv2)", RFC 1903, January 1996.

[7] SNMPv2 Working Group, Case, J., MCCloghrie, K., Rose, M. and S. Waldbusser, "Conformance Statements for Version 2 of the Simple Network Management Protocol (SNMPv2)", RFC 1904, January 1996.

[8] Case, J., Fedor, M., Schoffstall, M. and J. Davin, "Simple Network Management Protocol", STD 15, RFC 1157, May 1990.

[9] SNMPv2 Working Group, Case, J., McCloghrie, K., Rose, M. and S. Waldbusser, "Introduction to Community-based SNMPv2", RFC 1901, January 1996.

[10] SNMPv2 Working Group, Case, J., McCloghrie, K., Rose, M. and S. Waldbusser, "Transport Mappings for Version 2 of the Simple Network Management Protocol (SNMPv2)", RFC 1906, January 1996. 
[11] Case, J., Harrington D., Presuhn R. and B. Wijnen, "Message Processing and Dispatching for the Simple Network Management Protocol (SNMP)", RFC 2272, January 1998.

[12] Blumenthal, U. and B. Wijnen, "User-based Security Model (USM) for version 3 of the Simple Network Management Protocol (SNMPV3)", RFC 2274, January 1998.

[13] SNMPv2 Working Group, Case, J., McCloghrie, K., Rose, M. and S. Waldbusser, "Protocol Operations for Version 2 of the Simple Network Management Protocol (SNMPv2)", RFC 1905, January 1996.

[14] Levi, D., Meyer, P. and B. Stewart, "SNMPv3 Applications", RFC 2273, January 1998 .

[15] Wijnen, B., Presuhn, R. and K. McCloghrie, "View-based Access Control Model (VACM) for the Simple Network Management Protocol (SNMP) ", RFC 2275, January 1998.

[16] SNMPv2 Working Group, Case, J., McCloghrie, K., Rose, M. and S. Waldbusser, "Management Information Base for version 2 of the Simple Network Management Protocol (SNMPv2)", RFC 1907, January 1996.

[17] Information processing systems - Open systems Interconnection, "Specification of Abstract Syntax Notation One (ASN.1)", International Organization for Standardization, Internation Standard 8824, December 1987.

[18] Information processing systems - Open systems Interconnection, "Specification of Basic Encoding Rules for Abstract Syntax Notation One (ASN.1)", International Organization for Standardization, Internation Standard 8825, December 1987.

[19] McCloghrie, K., Heinanen, J., Greene, W. and A. Prasad, "Accounting Information for ATM Networks", RFC 2512, February 1999.

[20] Noto, M., Spiegel, E., and K. Tesink, "Definitions of Textual Conventions and OBJECT-IDENTITIES for ATM Management", RFC 2514, February 1999 . 


\section{Security Considerations}

The MIB defined in this memo controls and monitors the collection of accounting data. Care should be taken to prohibit unauthorized access to this control capability in order to prevent the disruption of data collection, possibly with fraudulent intent. Example of such disruption are disabling the collection of data, or causing the wrong set of data items to be collected.

SNMPv1 by itself is not a secure environment. Even if the network itself is secure (for example by using IPSec), even then, there is no control as to who on the secure network is allowed to access and GET/SET (read/change/create/delete) the objects in this MIB.

It is recommended that the implementers consider the security features as provided by the SNMPv3 framework. Specifically, the use of the User-based Security Model RFC 2274 [12] and the View-based Access Control Model RFC 2275 [15] is recommended.

It is then a customer/user responsibility to ensure that the SNMP entity giving access to an instance of this MIB, is properly configured to give access to the objects only to those principals (users) that have legitimate rights to indeed GET or SET (change/create/delete) them.

8. IANA Considerations

Prior to publication of this memo as an RFC, IANA is requested to make a suitable OBJECT IDENTIFIER assignment. 


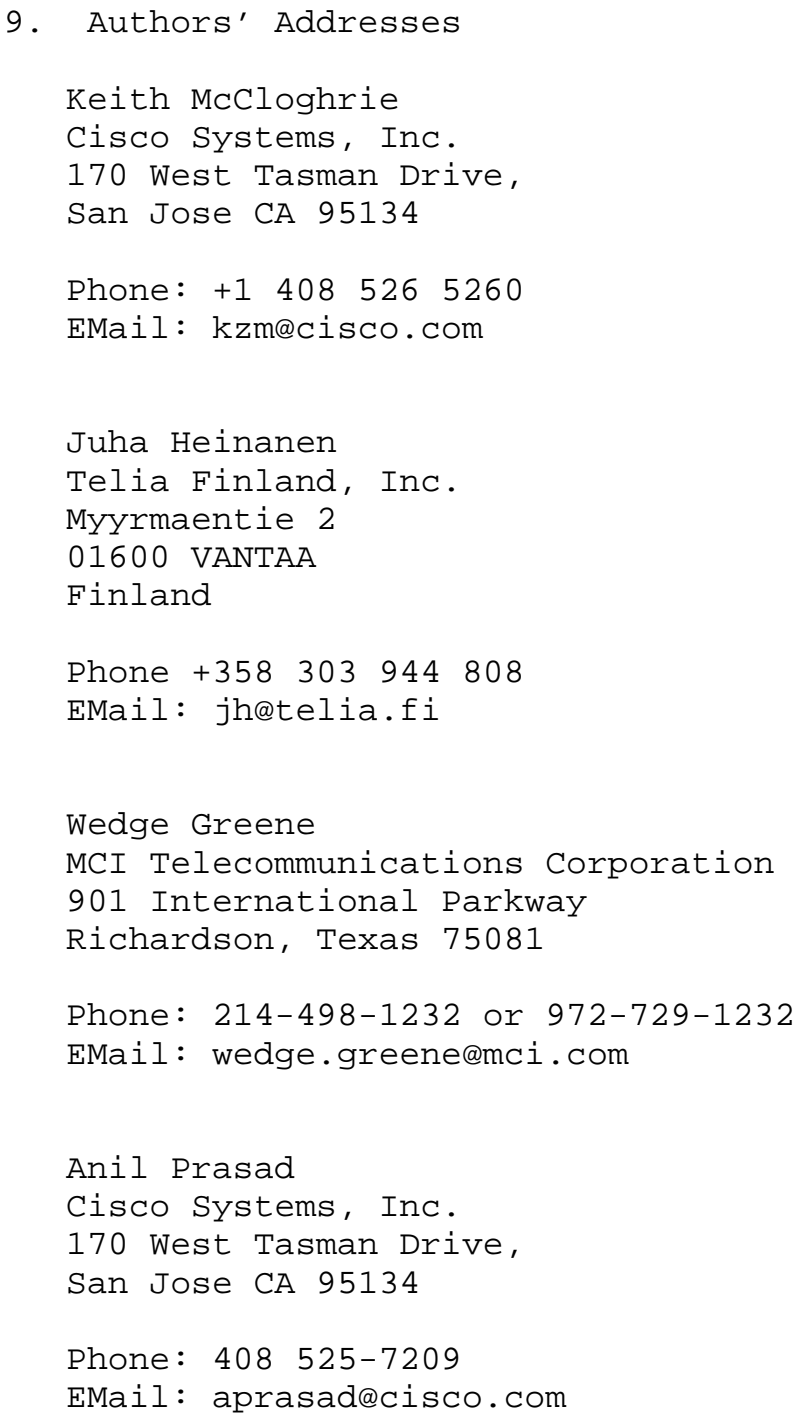


10. Full Copyright statement

Copyright (C) The Internet Society (1999). All Rights Reserved.

This document and translations of it may be copied and furnished to others, and derivative works that comment on or otherwise explain it or assist in its implementation may be prepared, copied, published and distributed, in whole or in part, without restriction of any kind, provided that the above copyright notice and this paragraph are included on all such copies and derivative works. However, this document itself may not be modified in any way, such as by removing the copyright notice or references to the Internet society or other Internet organizations, except as needed for the purpose of developing Internet standards in which case the procedures for copyrights defined in the Internet Standards process must be followed, or as required to translate it into languages other than English.

The limited permissions granted above are perpetual and will not be revoked by the Internet society or its successors or assigns.

This document and the information contained herein is provided on an "AS IS" basis and THE INTERNET SOCIETY AND THE INTERNET ENGINEERING TASK FORCE DISCLAIMS ALL WARRANTIES, EXPRESS OR IMPLIED, INCLUDING BUT NOT LIMITED TO ANY WARRANTY THAT THE USE OF THE INFORMATION HEREIN WILL NOT INFRINGE ANY RIGHTS OR ANY IMPLIED WARRANTIES OF MERCHANTABILITY OR FITNESS FOR A PARTICULAR PURPOSE. 